\title{
Performance analysis of data transmission in MC-CDMA radio interface with turbo codes
}

\author{
Andrzej Marczak
}

Published online: 10 December 2014

(C) The Author(s) 2014. This article is published with open access at Springerlink.com

\begin{abstract}
Multi-carrier code division multiple access (MCCDMA) technique is a combination of two radio access techniques: CDMA and orthogonal frequency division multiplexing and has the advantages of both techniques. The paper presents the design of transmitter and receiver for MC-CDMA radio interface. It also presents encoders and decoders of turbo codes which were used in simulation of the MC-CDMA technique. Two turbo codes with 8-state recursive systematic convolutional were used in the simulation. The simulation results of the transmission quality in additive white Gaussian noise (AWGN) channel presented in the paper show bit error rate and frame error rate performance of MC-CDMA technique with the turbo codes and allow for comparison the performance of both turbo codes.
\end{abstract}

Keywords MC-CDMA - Turbo codes - SOVA algorithm

\section{Introduction}

The three types of new multiple access schemes based on a combination of code division and orthogonal frequency division multiplexing (OFDM) techniques were proposed in 1993. These there types were "multi-carrier code division multiple access (MC-CDMA)", "multicarrier direct sequence CDMA" and "multitone (MT-) CDMA". The range of techniques have been presented in the literature $[4,7-$ $9,16]$.

The DS-CDMA technique applies spreading sequences in the time domain and uses Rake receiver to reduce effects

\section{A. Marczak (凶)}

Faculty of Electronics, Telecommunications and Informatics, Gdansk University of Technology, 11/12 Gabriela Narutowicza Street,

80-233 Gdańsk, Poland

e-mail: amarczak@eti.pg.gda.pl of fading in the radio channel. In MC-CDMA we can apply spreading sequences in the frequency domain, mapping a different chip of a spreading sequence to an individual OFDM subcarrier [7,10,12]. The signals of MC-CDMA can be easily transmitted and received using the fast Fourier transform (FFT) device without increasing the transmitter and receiver complexities. The signals have the attractive feature of high spectral efficiency due to minimally subcarrier spacing $[3,8]$.

\section{Construction if MC-CDMA transmitters}

The basic MC-CDMA signal is generated by a serial concatenation of classical DS-CDMA and OFDM. The transmitted signal of the $i$-th data symbol of the $j$-th user $s_{i}^{j}(t)$ is written as [7]:

$s_{i}^{j}(t)=\sum_{k=0}^{N-1} d_{i}^{j} c_{k}^{j} e^{2 \pi\left(f_{0}+k f_{d}\right) t} p(t-i T)$

where

$N \quad$ the number of subcarriers,

$d_{i}^{j} \quad$ the $i$-th message symbol of the $j$-th user,

$c_{k}^{j} \quad$ represents the $k$-th chip, $k=0, \ldots, N-1$, of the spreading sequence of the $j$-th user,

$f_{0} \quad$ the lowest subcarrier frequency,

$f_{d}$ the subcarrier separation,

$p(t)$ a rectangular signaling pulse shifted in time given by [7]:

$p(t)= \begin{cases}1 & \text { for } 0 \leq t \leq T \\ 0 & \text { otherwise }\end{cases}$ 


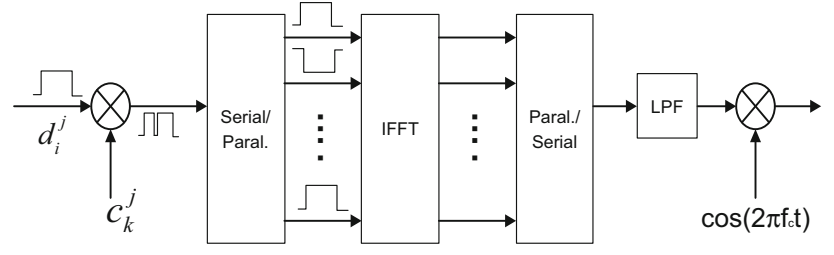

Spreader OFDM Modulator

Fig. 1 Diagram of MC-CDMA transmitter [7]

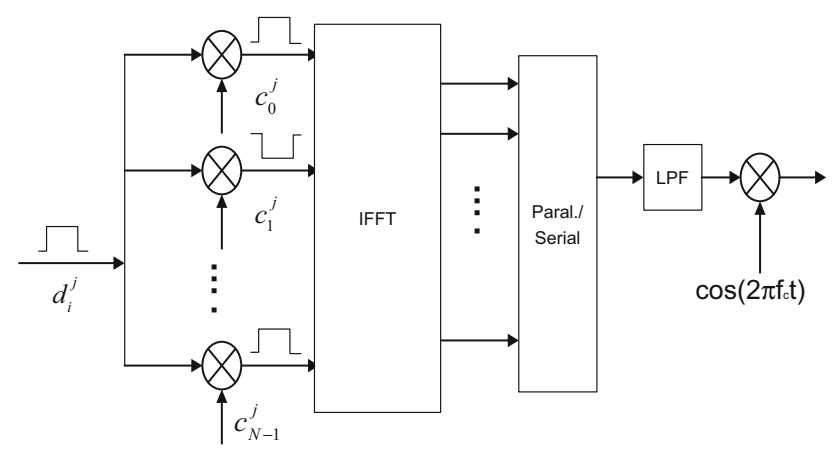

Fig. 2 Diagram of alternative MC-CDMA transmitter [7]

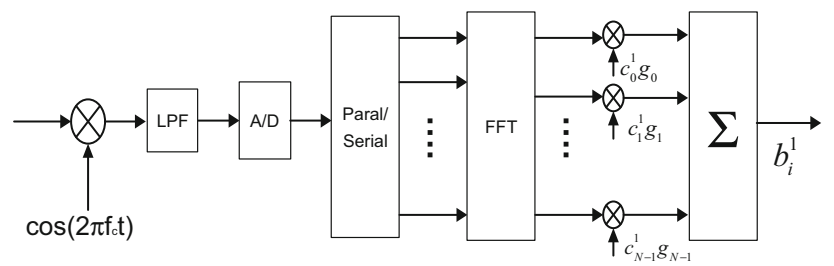

Fig. 3 Diagram of MC-CDMA receiver [7]

The transmitter structure can be implemented by concatenating a DS-CDMA spreader and an OFDM transmitter as shown in Fig. 1. The information bit, $d_{i}^{j}$ is spread in the time domain. In this implementation, high speed operations are required at the output of the spreader. The spread chips are fed into the serial to parallel (Serial/Parall.) block and inverse fast Fourier transform (IFFT) is applied to these $N$ parallel chips. The values at the output of the IFFT are time domain samples in parallel form. The samples after parallel to serial conversion (Parall./Serial) are low pass filtered in order to obtain the continuous time domain signal. The signal modulates the carrier and is transmitted to the receiver [7].

Another implementation is shown in Fig. 2. The time domain spreader is removed. In this implementation, the spreading sequence is applied directly to the identical parallel input bits. The high speed spreading operation is not required in the implementation [8]. The spreading sequences in MC-CDMA separate other users' signal from desired signal, provided that their spreading sequences are orthogonal to each other [7].

At the receiver of MC-CDMA technique (Fig. 3) each carrier's symbol, the corresponding chip $c_{k}^{j}$ of user $j$, is recovered using FFT after sampling and the recovered chip sequence is correlated with desired user's spreading code in order to recover the original information $d_{i}^{j}$. Can be defined the $i$-th received symbol at $k$-th carrier as [7]:

$r_{k, i}=\sum_{j=0}^{J-1} H_{k} d_{i}^{j} c_{k}^{j}+n_{k, i}$

where

$J$ the number of users,

$H_{k}$ the frequency response of the $k$-th subcarrier,

$n_{k}$ the corresponding noise sample.

The MC-CDMA receiver of the 1-th user multiplies received symbol rs by its spreading sequence chip $c_{k}^{1}$, as well as by the gain $g_{k}$, which is given by the reciprocal of the estimated channel transfer factor of subcarrier $k$, for each received subcarrier symbol $k=0, \ldots, N-1$. Then the receiver sums all these products in order to arrive at the decision variable $b_{i}^{1}$ [7]:

$b_{i}^{1}=\sum_{k=0}^{N-1} c_{k}^{1} g_{k} r_{k, i}$

\section{Construction of turbo encoders and decoder}

Two turbo encoders were used in the simulations. The first turbo encoder was the standard turbo encoder from the UMTS. The turbo encoder has coding rate $1 / 3$ and is composed of two 8-state, identical, parallel, recursive, systematic convolutional (RSC) encoders and the internal interleaver (Fig. 4) [6].

The second turbo encoder has the same coding rate $(1 / 3)$ and is composed of two 8-state identical RSC encoders [14]. The diagram of second turbo encoder is shown in Fig. 5.

In both turbo encoders, interleaving is executed, in a rectangular array $[1,6,11,13]$. Its main task is to reduce the

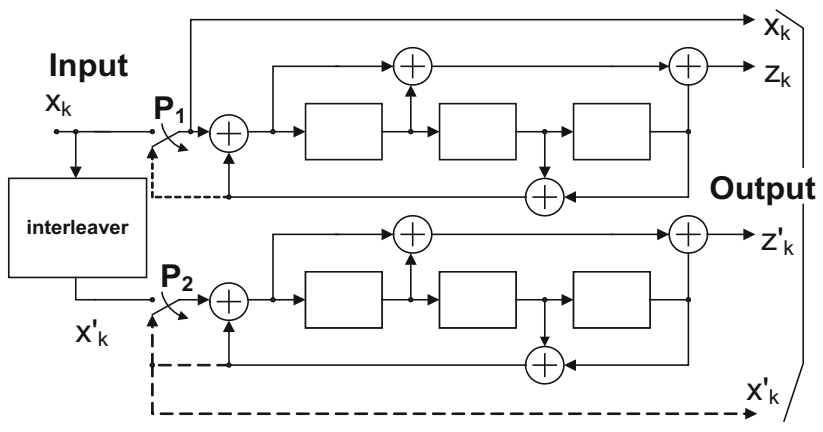

Fig. 4 Diagram of the first turbo encoder [6] 


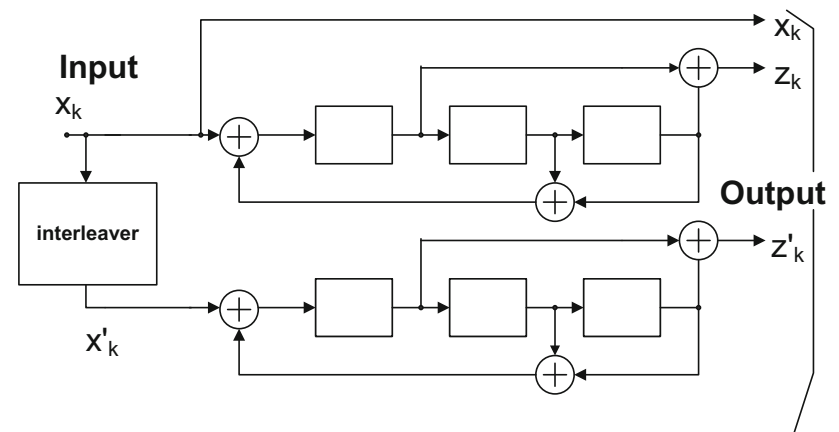

Fig. 5 Diagram of the second turbo encoder [14]

degree of statistical relationships between input data of the upper and lower convolutional encoders in a turbo encoder [15]. The number of bits $K$ fed to the inputs of convolutional encoders in the turbo encoder may be in the range from 40 to 5,114 bits.

Coding sequences are formed in the turbo encoder by taking a sequence of bits: the systematic output $\mathrm{x}_{\mathrm{k}}$, the output of the upper encoder $z_{k}$, and output the lower encoder $z_{k}^{\prime}[6]$.

The turbo decoder consists of two soft output convolutional code decoders (SOVA decoder 1 and SOVA decoder 2), two interleavers, two deinterleavers and decision-making unit at the output of the decoder (Fig. 6) [11]. Interleaver units, in the turbo decoder are identical with the internal interleaver in turbo encoder. Deinterleavers perform the inverse operation, which restores the original order of data bits. Decision-making unit produces binary values from soft values at the output of convolutional code decoder (SOVA decoder 2).

The convolutional code decoders in the turbo decoder can use algorithms based on the maximum a posteriori probability rule (MAP) [2]. In addition to these uses the Soft Output Viterbi Algorithm (SOVA) [11]. The SOVA is a modified version of the Viterbi algorithm.

Decoding turbo codes is an iterative process. It consists of separate decoding received sequences from both convolutional encoders of turbo encoder [15]. The SOVA decoder 1 decodes the sequence received from the upper encoder, $\mathrm{z}_{\mathrm{k}}$, and the SOVA decoder 2-the sequence received from the lower encoder $z_{k}^{\prime}$. The first SOVA decoder uses the extrinsic information $\mathrm{L}_{\mathrm{e} 2}$ from the second SOVA decoder in decoding process. The extrinsic information includes a priori probability of the information bits [14]. Similarly, the second SOVA decoder uses the extrinsic information $\mathrm{L}_{\mathrm{e} 1}$ derived from first SOVA decoder [11].

\section{Simulation results}

The simulation software of the MC-CDMA technique, used in the research allows the testing of transmission quality for two turbo codes, several values of spreading factor $(\mathrm{SF}=8$, 32,64 and 128) and data bits number $(K=640$ and 1,440$)$. The first turbo encoder (turbo code 1) design is based on the diagram in Fig. 4 and the second turbo encoder (turbo code 2) design is based on the diagram in Fig. 5. The MC-CDMA transmitter design is based on the schema in Fig. 1 and the receiver design is based on the schema in Fig. 3.

Figures $7,8,9,10,11,12,13$, and 14 show the results of transmission quality (bit error rate-BER and frame error rate-FER) as a function of $\mathrm{E}_{\mathrm{b}} / \mathrm{N}_{0}$ for MC-CDMA technique with first turbo code (turbo code 1). Figure 7 shows the BER of MC-CDMA for spreading factor $\mathrm{SF}=8$ and number of data bits $K=640$ and 1,440 bits. For larger data frame length $(\mathrm{K}=1,440)$ the same quality of transmission was achieved for the ratio $\mathrm{E}_{\mathrm{b}} / \mathrm{N}_{0}$ by approximately $0.2 \div 0.5 \mathrm{~dB}$ lower than the data frame with $\mathrm{K}=640$ bits. Figure 8 shows the FER of MC-CDMA for the same SF and K. In this graph you may notice that for the value of $\mathrm{E}_{\mathrm{b}} / \mathrm{N}_{0}=3 \mathrm{~dB}$, for $\mathrm{K}=1,440$ bits FER is almost five times lower than for $\mathrm{K}=640$ bits.

Similar results can be observed also for other values of SF (Figs. 9, 10, 11, 12, 13, 14). Comparing the transmission quality results for the same K, but different values of SF can be seen to improve transmission quality while increasing the value of SF. The best transmission quality was achieved for $\mathrm{SF}=128$ and $\mathrm{K}=1,440$ bits $\left(\mathrm{BER}=10^{-6}\right.$ for $\mathrm{E}_{\mathrm{b}} / \mathrm{N}_{0}=$ $2.9 \mathrm{~dB}$ ). In Figs. 13 and 14 you may notice that for the value of
Fig. 6 Structure of turbo decoder

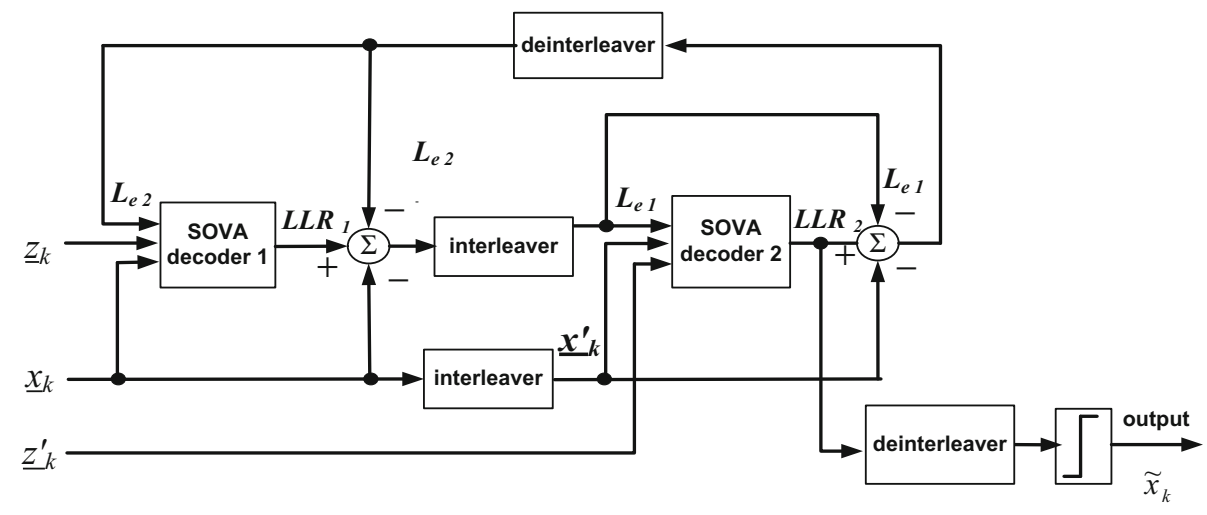




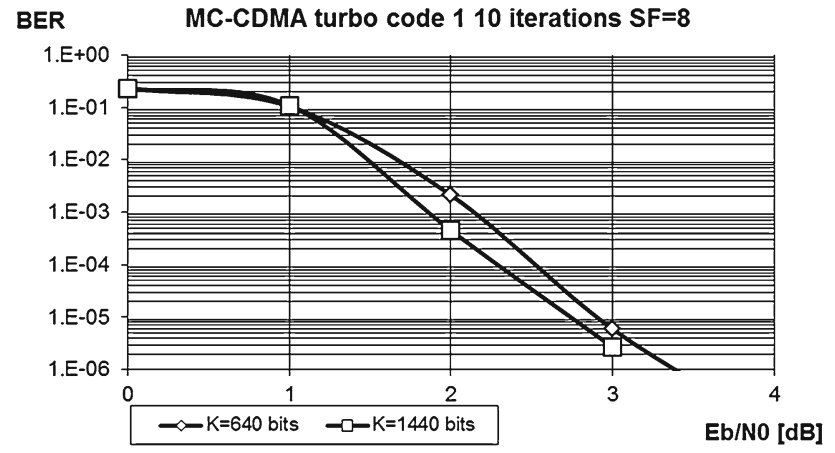

Fig. 7 BER performance of MC-CDMA with turbo code $1(\mathrm{SF}=8)$

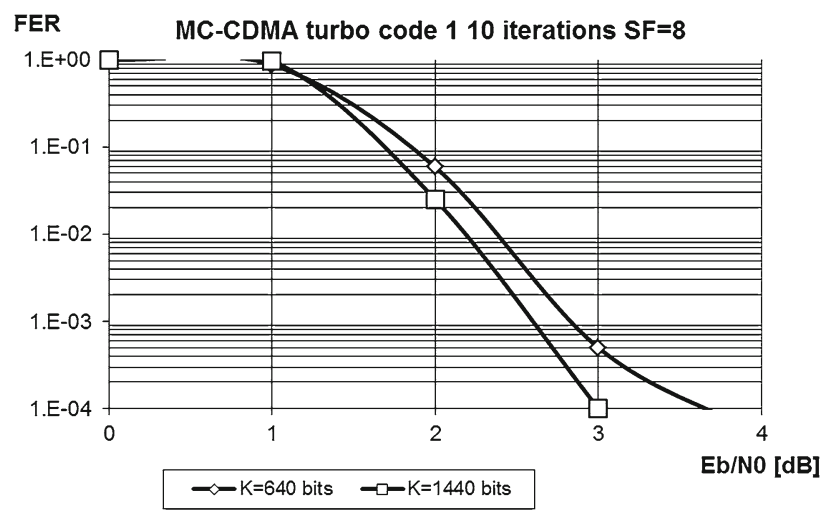

Fig. 8 FER performance of MC-CDMA with turbo code $1(\mathrm{SF}=8)$

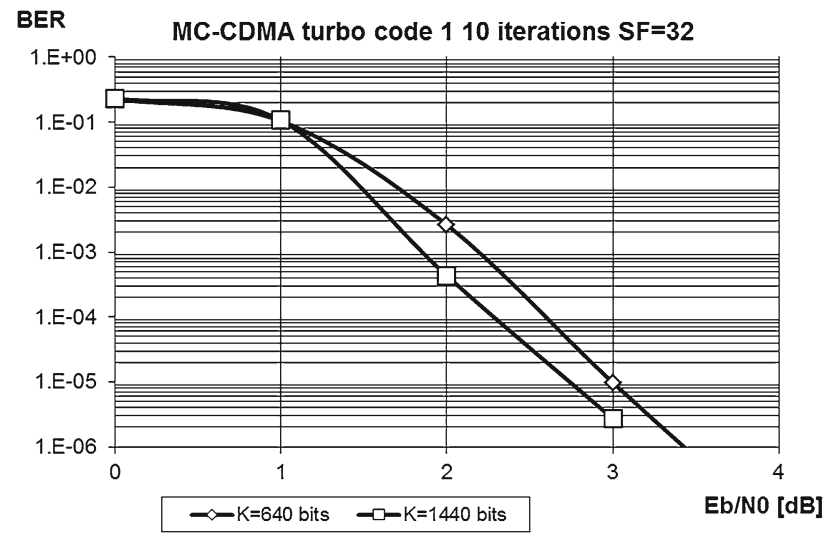

Fig. 9 BER performance of MC-CDMA with turbo code $1(\mathrm{SF}=32)$

$\mathrm{E}_{\mathrm{b}} / \mathrm{N}_{0}=2.9 \mathrm{~dB}$, for $\mathrm{K}=1,440$ bits, BER and FER is almost 20 times lower than for $\mathrm{K}=640$ bits.

Figures 15, 16, 17, 18, 19, 20, 21, and 22 show the results of transmission quality as a function of $\mathrm{E}_{\mathrm{b}} / \mathrm{N}_{0}$ for MCCDMA technique with the second turbo code (turbo code 2). Figure 15 shows the BER of MC-CDMA for spreading factor $\mathrm{SF}=8$ and number of data bits $\mathrm{K}=640$ and 1,440 bits. For larger data frame length $(\mathrm{K}=1,440)$ the same quality of transmission was achieved for the ratio $\mathrm{E}_{\mathrm{b}} / \mathrm{N}_{0}$ by approximately $0.2 \mathrm{~dB}$ lower than the data frame with $\mathrm{K}=640$ bits.

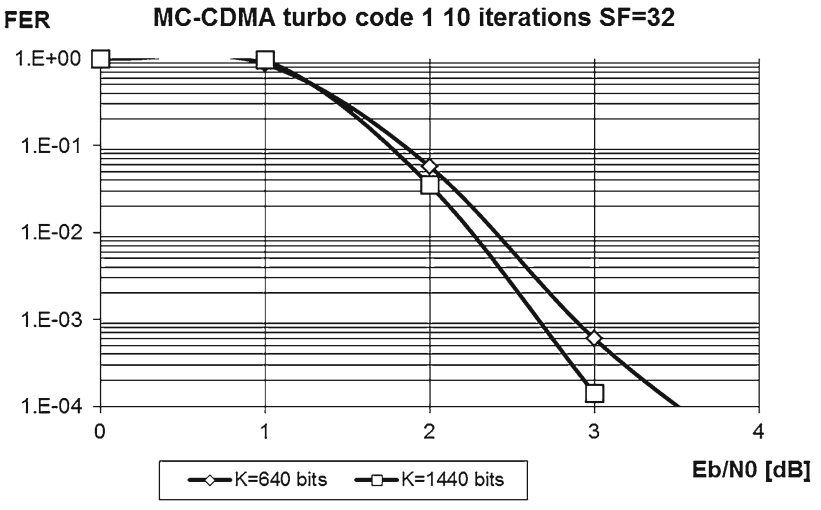

Fig. 10 FER performance of MC-CDMA with turbo code $1(\mathrm{SF}=32)$

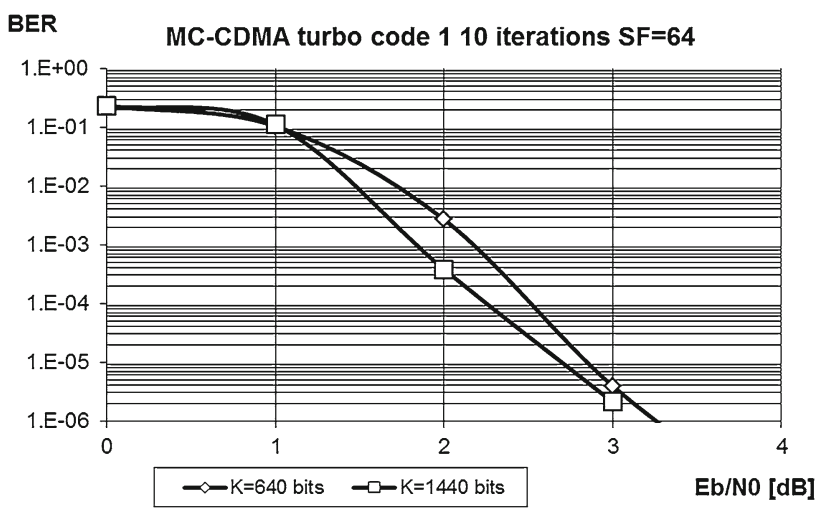

Fig. 11 BER performance of MC-CDMA with turbo code $1(\mathrm{SF}=64)$

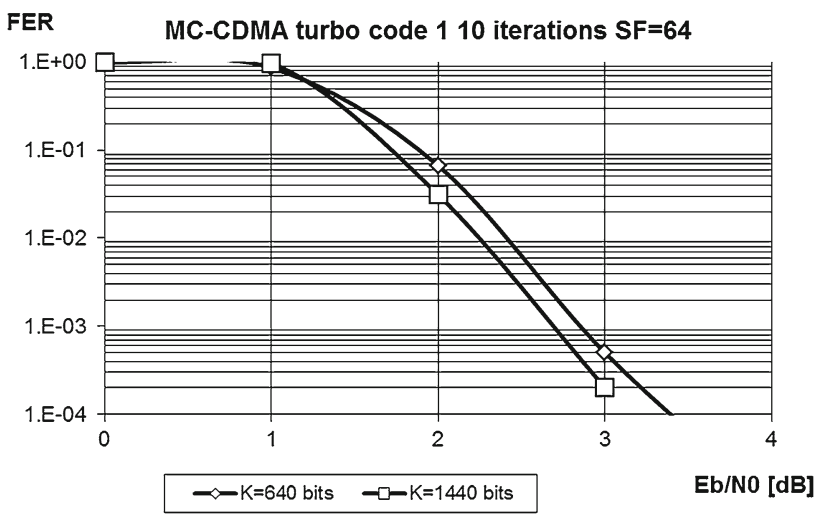

Fig. 12 FER performance of MC-CDMA with turbo code $1(\mathrm{SF}=64)$

Figure 16 shows the FER of MC-CDMA for the same SF and $\mathrm{K}$. In this graph you may notice that FER for the value of $\mathrm{E}_{\mathrm{b}} / \mathrm{N}_{0}=4 \mathrm{~dB}$, for $\mathrm{K}=1,440$ bits and for $\mathrm{K}=640$ bits is almost equal $\left(7 \times 10^{-6}\right.$ for $\mathrm{K}=1,440$ and $8 \times 10^{-6}$ for $\mathrm{K}=640$ ). The best transmission quality was achieved for $\mathrm{SF}=128$ and $\mathrm{K}=1,440$ bits. For larger data frame length $(\mathrm{K}=1,440)$ the same quality of transmission was achieved for the ratio $\mathrm{E}_{\mathrm{b}} / \mathrm{N}_{0}$ by approximately $1 \mathrm{~dB}$ lower than the data frame with $\mathrm{K}=640$ bits (Fig. 21). 


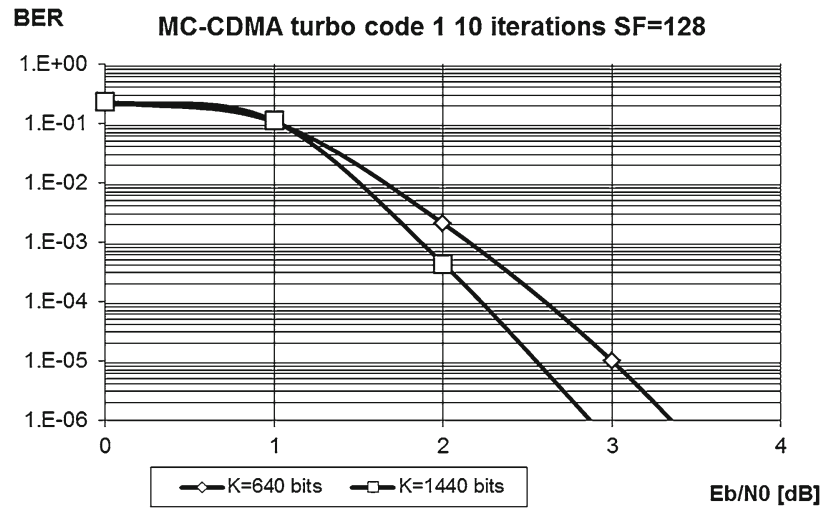

Fig. 13 BER performance of MC-CDMA with turbo code $1(\mathrm{SF}=128)$

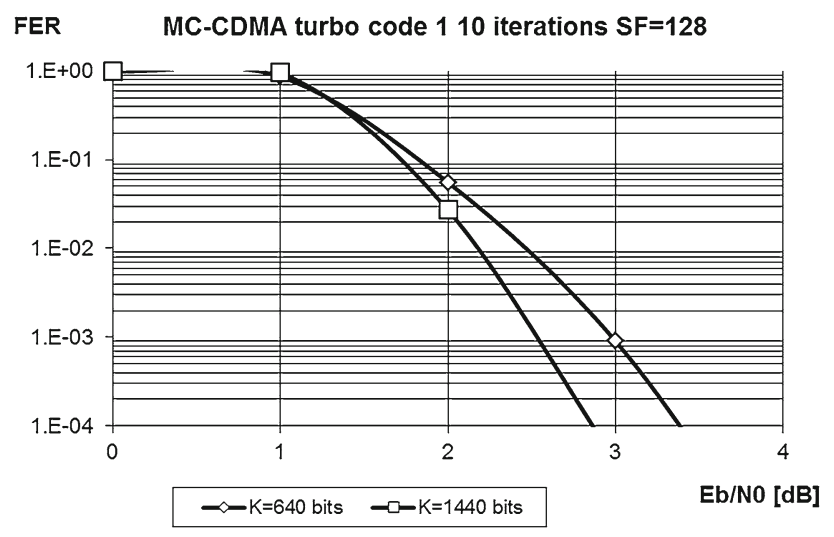

Fig. 14 FER performance of MC-CDMA with turbo code $1(\mathrm{SF}=128)$

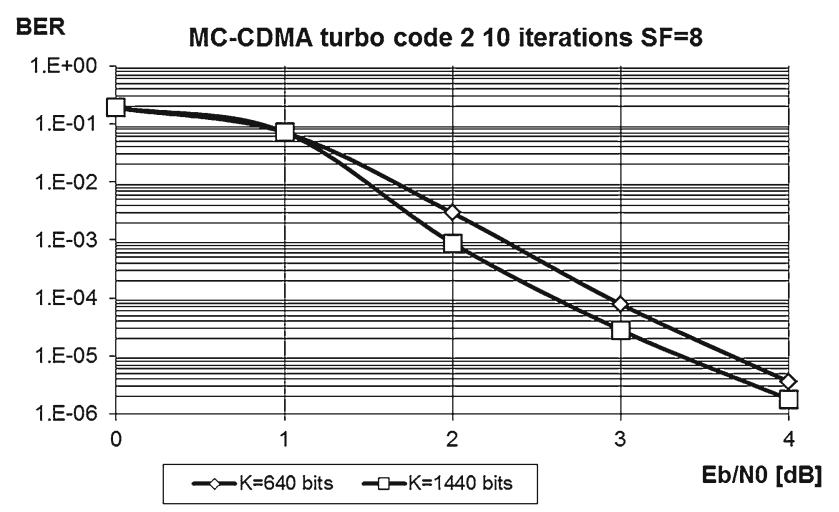

Fig. 15 BER performance of MC-CDMA with turbo code $2(\mathrm{SF}=8)$

Figures 23, 24, 25, and 26 show the results of transmission quality as a function of $\mathrm{E}_{\mathrm{b}} / \mathrm{N}_{0}$ for MC-CDMA technique for the both turbo codes for $\mathrm{SF}=128$ and $\mathrm{K}=640$ and 1,440 bits.

Based on Figs. 23 and 25 you can compare results of BER for both turbo codes and $\mathrm{SF}=128$. If the value of $\mathrm{E}_{\mathrm{b}} / \mathrm{N}_{0}$ was smaller then $2 \mathrm{~dB}$ (for $\mathrm{K}=640$ ) and $1.5 \mathrm{~dB}$ (for $\mathrm{K}=1,440$ ) the better results of BER was achieved for turbo code 2 . For other values of $E_{b} / N_{0}$ you may notice the better results of BER for turbo code 1 .

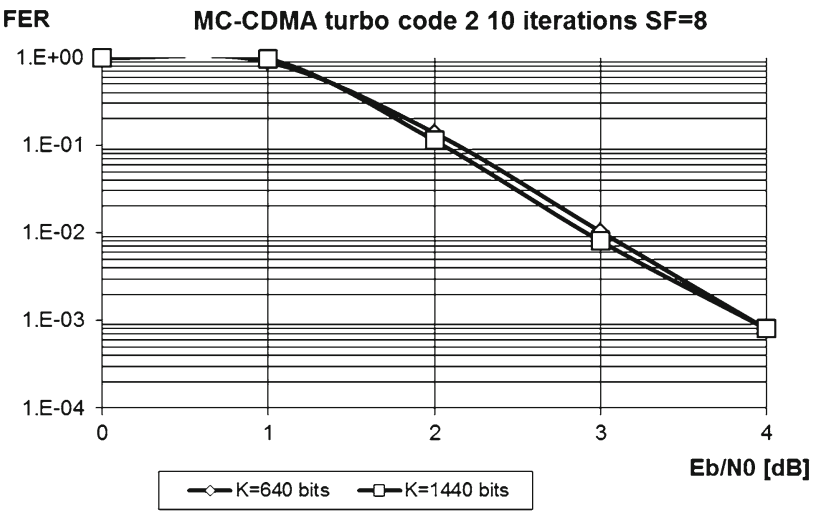

Fig. 16 FER performance of MC-CDMA with turbo code $2(\mathrm{SF}=8)$

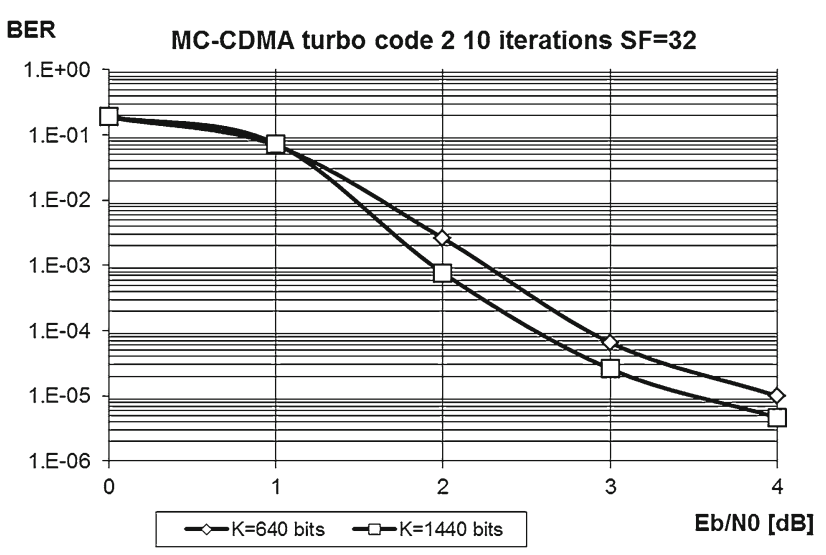

Fig. 17 BER performance of MC-CDMA with turbo code $2(\mathrm{SF}=32)$

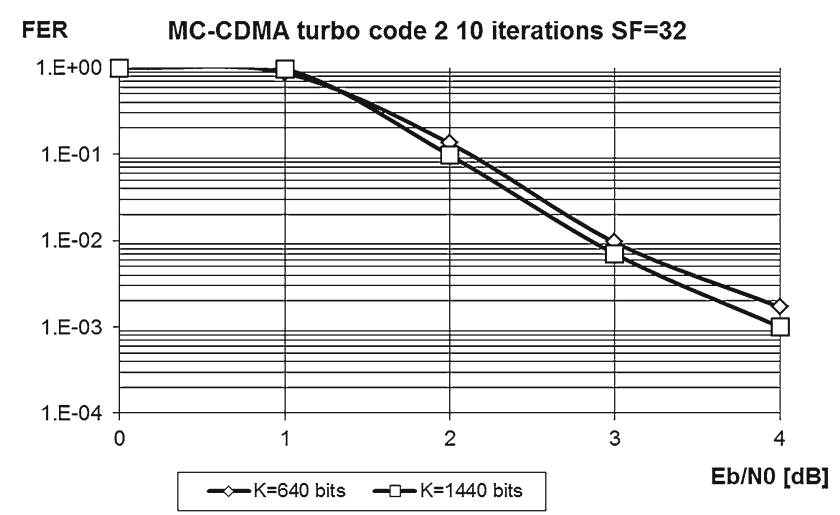

Fig. 18 FER performance of MC-CDMA with turbo code $2(\mathrm{SF}=32)$

Based on Figs. 24 and 26 you can compare results of FER for both turbo codes and $\mathrm{SF}=128$. If the value of $\mathrm{E}_{\mathrm{b}} / \mathrm{N}_{0}$ was greater then $1 \mathrm{~dB}$ (for both $\mathrm{K}$ ) the better results of FER was achieved for turbo code 1 . 


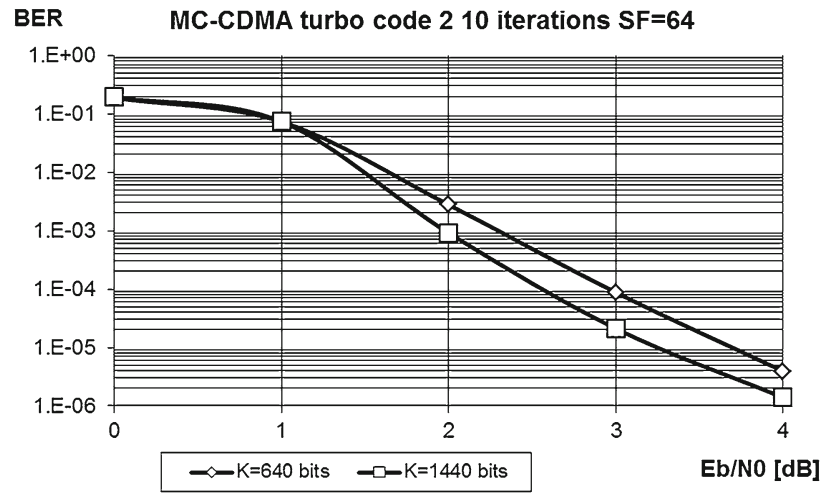

Fig. 19 BER performance of MC-CDMA with turbo code $2(\mathrm{SF}=64)$

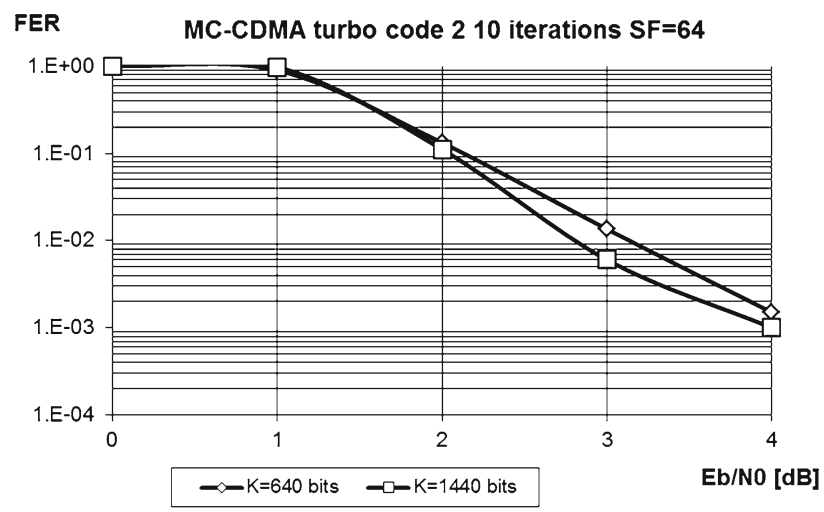

Fig. 20 FER performance of MC-CDMA with turbo code $2(\mathrm{SF}=64)$

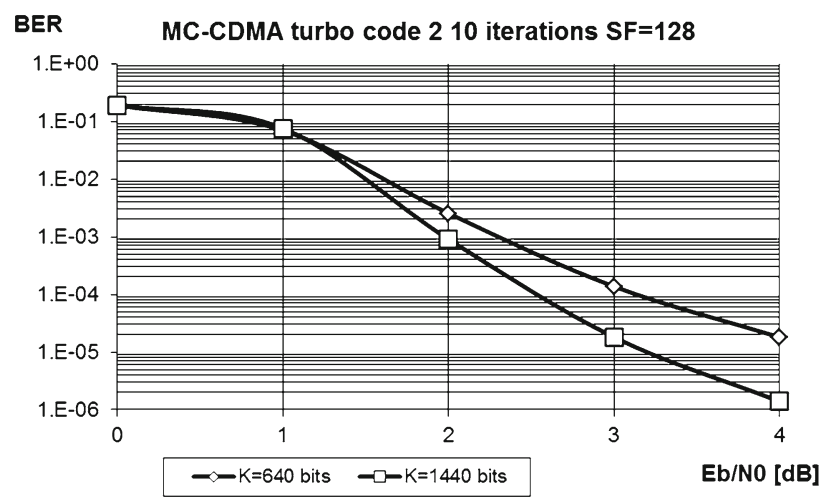

Fig. 21 BER performance of MC-CDMA with turbo code $2(\mathrm{SF}=128)$

\section{Conclusion}

The research results allow us to assess the usefulness of turbo codes in the MC-CDMA technique. The use of turbo coding in the radio interface allows for high quality transmission, which is presented on the results of bit and frame error rates. The presented simulation results correspond to the situation in which a single connection is performed between the transmitter and the receiver. In real conditions, the problem of system load, interference from other users, and throughput-

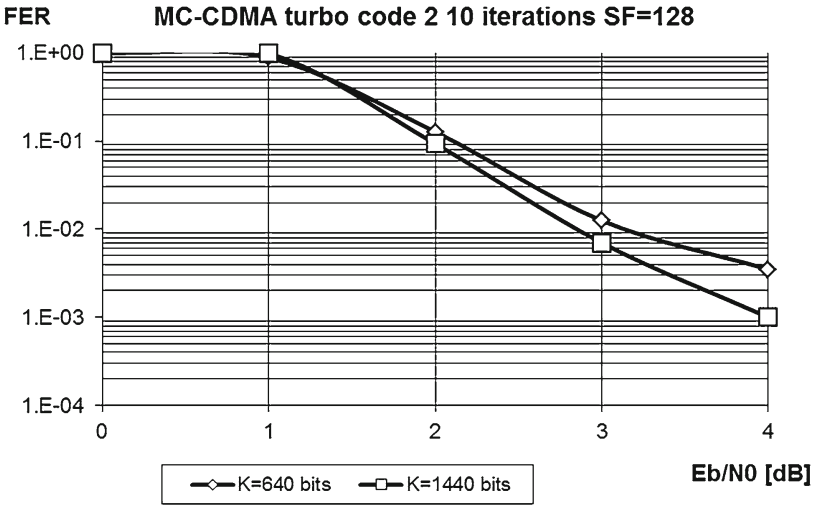

Fig. 22 FER performance of MC-CDMA with turbo code $2(\mathrm{SF}=128)$

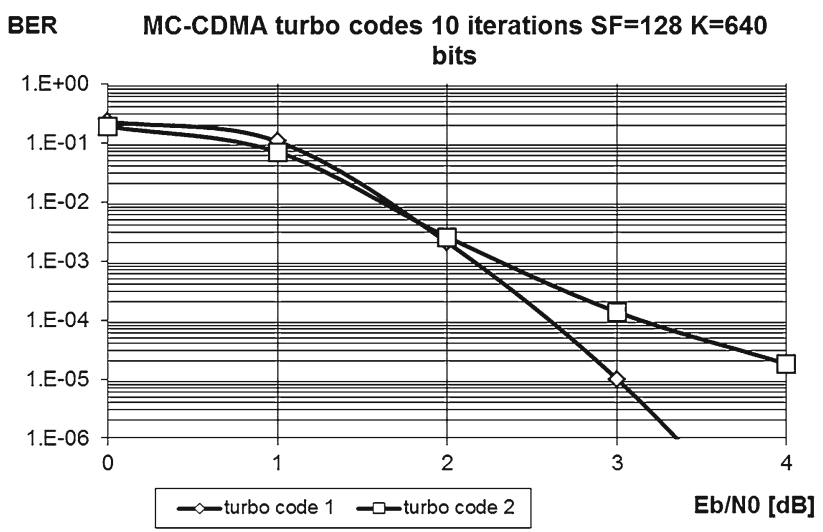

Fig. 23 BER performance of MC-CDMA with turbo codes $(\mathrm{SF}=128$, $\mathrm{K}=640$ )

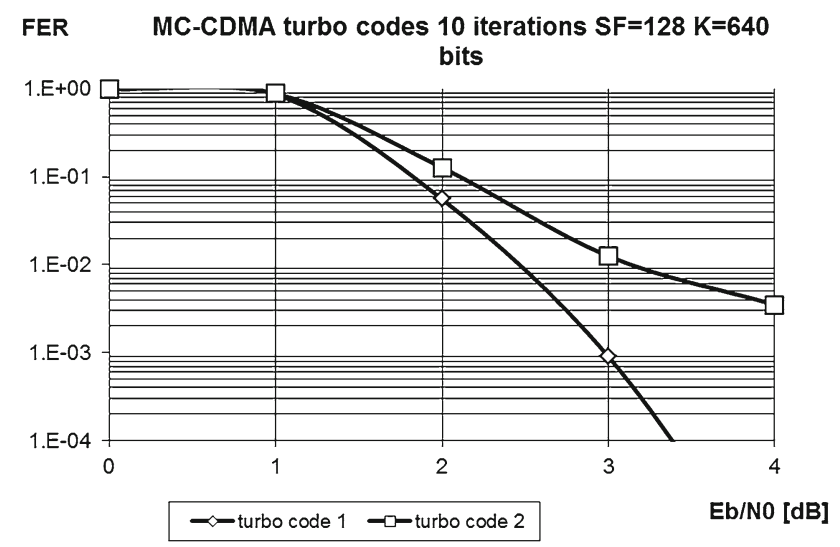

Fig. 24 FER performance of MC-CDMA with turbo codes $(\mathrm{SF}=128$, $\mathrm{K}=640$ )

coverage characteristics of the system can be significant [5], due to the specificity of the CDMA system, what is also important for MC-CDMA. The best transmission quality results $\left(\mathrm{BER}=10^{-6}\right.$ and $\left.\mathrm{FER}=10^{-4}\right)$ were achieved for $\mathrm{SF}=128$ and $\mathrm{K}=1,440$ bits for the turbo code 1 . For the turbo code 2 simulation results are worse $\left(\mathrm{BER}=10^{-6}\right.$ and FER $=10^{-3}$ ). Although the trellises of RSC in both turbo 


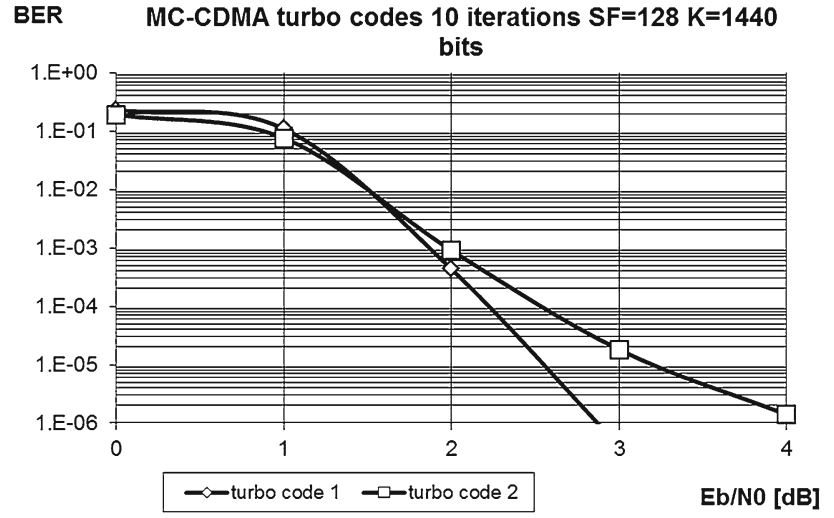

Fig. 25 BER performance of MC-CDMA with turbo codes ( $\mathrm{SF}=128$, $\mathrm{K}=1,440)$

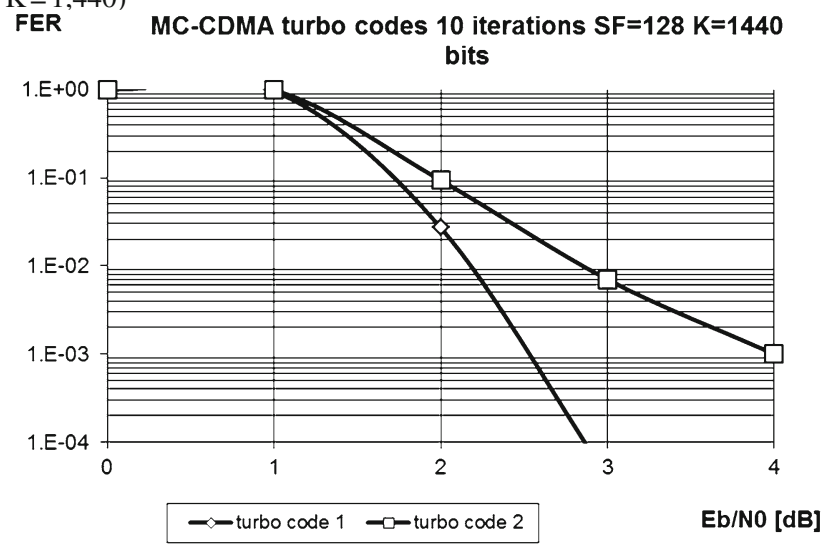

Fig. 26 FER performance of MC-CDMA with turbo codes $(\mathrm{SF}=128$, $\mathrm{K}=1,440$ )

codes has 8 states, you may notice the better performance of turbo code 1 which was used in UMTS. The obtained results confirm suitability of turbo coding for use in radio communication systems, especially realizing high-speed data services, where data bits number $\mathrm{K}$ is greater than 1,000 bits. In this case the efficiency of turbo codes and the MC-CDMA techniques is the best. If the data bits number is less than 1,000 bits the efficiency is worse and can be proposed to replace the turbo code by the convolutional code in the MC-CDMA technique due to the computational complexity of decoding the turbo codes.

Open Access This article is distributed under the terms of the Creative Commons Attribution License which permits any use, distribution, and reproduction in any medium, provided the original author(s) and the source are credited.

\section{References}

1. Benedetto, S., \& Montosi, G. (1996). Unveiling turbo codes: Some results on parallel concatenated coding schemes. IEEE Transactions on Information Theory, 42(2), 409-428.

2. Berrou, C., \& Glavieux, A. (1996). Near optimum error correcting coding and decoding: Turbo-codes. IEEE Transactions on Communications, 44(10), 1261-1271.
3. Cariou, L., \& Helard, J. F. (2005). MIMO frequency hopping spread spectrum multi-carrier multiple access: A novel uplink system for B3G cellular networks. Telecommunication Systems, 30(1-3), 193 214.

4. Enayati, A. R., Azmi, P., Taghinia, Y., \& Salahi, A. (2012). A novel bandwidth efficient SOC-based turbo coding scheme mid reduced complexity MUD for SA-based MC-CDMA systems. Telecommunication Systems,. doi:10.1007/s11235-010-9389-9.

5. Gajewski, S. (2009). A method of the UMTS-FDD network design based on universal load characteristics. In J. Woznaik \& J. Konorski (Eds.), Wireless and mobile networking, proceedings book series: IFIP advances in information and communication technology (Vol. 308, pp. 274-285).

6. GPP TS 25.212 v9.0.0 3rd Generation Partnership Project; Technical Specification Group Radio Access Network; "Multiplexing and channel coding (FDD)".

7. Hanzo, L., Münster, M., Choi, B. J., \& Keller, T. (2003). OFDM and MC-CDMA for broadband multi-user communications, WLANs and broadcasting. Chichester: Wiley.

8. Hara, S., \& Prasad, R. (1997). Overview of multicarrier CDMA. IEEE Communications Magazine, 35(12), 126-133.

9. Kaiser, S., \& Fazel, K. (2003). Multi-carrier and spread spectrum systems. Chichester: Wiley.

10. Maity, S. P., Maity, S., \& Sil, J. (2012). Multicarrier spread spectrum watermarking for secure error concealment in fading channel. Telecommunication Systems. doi:10.1007/s11235-010-9369-0.

11. Marczak, A. (2011). Comparison of the efficiency of turbo codes with different number of states. In Proceeding 34th international conference on telecommunications and signal processing (TSP), Budapest.

12. Sacchi, C., \& Panizza, M. (2011). Multi-rate group-orthogonal OFDMA-CDMA for broadband mobile transmission. Telecommunication Systems. doi:10.1007/s11235-011-9441-4.

13. Scalise, S., Ernst, H., \& Ko Bae, Y. J. (2003). A fast algorithm to estimate the distance spectrum of turbo codes and tail-biting turbo codes. Telecommunication Systems, 24(2-4), 167-185.

14. Sklar, B. (1997). A primer on turbo code concepts. IEEE Communications Magazine, 35(12), 94-102.

15. Vucetic, B., \& Yuan, J. (2000). Turbo codes principles and applications. Boston, MA: Kluwer Academic Publishers.

16. Yee, N., \& Linnartz, J. P. Multi-carrier code division multiple access (MC-CDMA): A new spreading technique for communication over multipath channels. Final report 1993-1994 for MICRO Project 93-101.

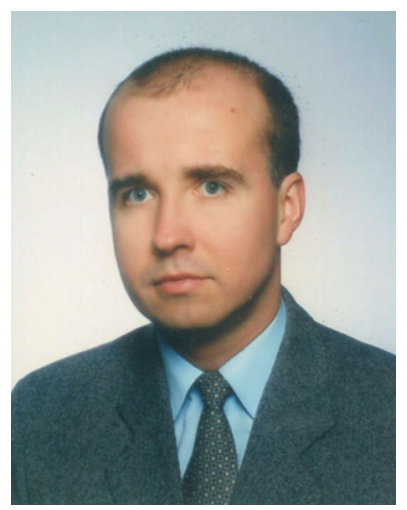

Andrzej Marczak was born in Gdynia (Poland). He obtained the Master and Ph.D. degrees of Telecommunication Engineering at the Gdansk University of Technology (Poland) in 1997 and 2005, respectively. Since February 2006 Dr. Marczak has been holding a position as assistant professor at the Faculty of Electronics, Telecommunications and Informatics of the Gdansk University of Technology. The research interests of Dr. Marczak are mainly focused on: software defined radio (SDR) technology, multi-access wireless communications (MC-CDMA, DS-CDMA, OFDMA), digital signal processing, channel coding techniques (turbo coding and LDPC coding) and data security techniques. 